Global Journal of Pure and Applied Mathematics.

ISSN 0973-1768 Volume 13, Number 10 (2017), pp. 7601-7625

(C) Research India Publications

https://dx.doi.org/10.37622/GJPAM/13.10.2017.7601-7625

\title{
Simulated Annealing with Clone Operator
}

\author{
G.S. Biju \\ Department of Mathematics, \\ College of Engineering, \\ Thiruvananthapuram, Kerala, India, 695016. \\ A.K. Anilkumar \\ Vikram Sarabhai Space Centre, \\ Indian Space Research Organisation, \\ Thiruvananthapuran, India.
}

\begin{abstract}
Simulated Annealing has been a very successful general algorithm for the solution of large, complex combinatorial optimization problems. In this paper, we propose a clone operator which imitates the biological cloning process to generate a candidate solution vector for the solution vector in simulated annealing algorithm. The proposed algorithms are tested with various case studies obtained from the literature and the results are presented. The algorithm is capable of getting optima.
\end{abstract}

\section{AMS subject classification:}

Keywords: simulated annealing, metaheuristic, multiobjective optimization, annealing schedule.

\section{Introduction}

Simulated annealing (SA) is a compact and robust randomized metaheuristic technique, which provides excellent solutions to single and multiple objective optimization problems with a substantial reduction in computation time. It is a proven method to obtain an optimal solution for a single objective optimization problem and to obtain a Pareto set of solutions for a multi-objective optimization problem. It is based on an analogy of thermodynamics with the way metals cool and anneal. If a liquid metal is cooled slowly, 
its atoms form a pure crystal corresponding to the state of minimum energy for the metal. The metal reaches a state with higher energy, if it is cooled quickly. SA has received a lot of attention in the recent times to solve optimization problems. Kirkpatrick et al. [1] and Cerny [2] showed a model for simulating the annealing of solids, based on the theory originally proposed by Metropolis et al. [3]. This model is used for the optimization of problems, where the objective functions to be minimized corresponds to the energy states of the metal. SA has these days become one of the many heuristic approaches designed to give a good, not necessarily optimal solution. Since its introduction, several applications in different fields of engineering, such as integrated circuit placement, VLSI design, pattern recognition, optimal encoding, resource allocation, logic synthesis, have been deploying SA solution. At the same time, theoretical studies have been focusing on the reasons for the excellent behavior of the algorithm. Theoretical developments and researches in simulated annealing are well documented ([4, 5, 6, 7, 8, 9, 10, 11]).

Though, the majority of the theoretical developments and application work with simulated annealing has been for discrete optimization problems, simulated annealing has also been used as a tool to address problems in the continuous domain. We are observing an increased interest in using simulated annealing for global optimization over regions containing several local and global minima. Fabian [12] studies the performance of simulated annealing methods for finding a global minimum of a given objective function. Bohachevsky et al. [13] proposes a generalized simulated annealing algorithm of function optimization for the use in statistical applications and Locatelli [14] presents a proof of convergence for the algorithm. Belisle [15] presents a special simulated annealing algorithm for global optimization, which uses a heuristically motivated cooling schedule. This algorithm provides a reasonable alternative to existing methods and can be implemented easily. Suman $([16,17,18])$ proposes different simulated annealingbased approach to address constrained multi-objective optimization problems. In [17], a comparative analysis of five simulated annealing algorithms is conducted for the system reliability optimization problem. These methods were aimed to generate a set of efficient solutions that are a good approximation to the entire set of efficient solutions over a fixed period of time.

Belisle et al. [19] discusses convergence properties of simulated annealing algorithms applied to continuous functions and apply these results to hit-and-run algorithms used in global optimization. The convergence properties identified are consistent with those presented in [20] and provide a good contrast between convergence in probability and almost sure convergence. This work is further extended in [21] to an improved hitand-run algorithm used for global optimization. Several researchers have independently proven the convergence results ([20, 22, 23, 24, 25, 26]).

In this paper, firstly, we give a description of simulated annealing algorithm. Section II gives a description of SA algorithm. In section III, a clone operator which imitates the biological cloning process to generate a candidate solution vector in simulated annealing is proposed. The computational results of test problems are shown in section IV. Finally, section V summarizes the concluding remarks. 


\section{Simulated Annealing}

The basic approach in SA considers the neighbouring state of the current state to reach a stable state for the application. The strategy consists of exploring the solution space starting from an arbitrary selected solution or state and generating a new one produced through conservatively modifying a given state. Every time a new solution is generated, its cost is evaluated to accept or reject the solution according to an acceptance rule. In SA, as we are looking for an actual best solution which would be a global optimum, the algorithm some times even accepts the worse move to avoid being trapped in a local minimum. The acceptance and rejection of the worse move is controlled by a probability function. The probability of accepting a move, which causes an increase $\Delta f$ in the objective function $f$, is called the acceptance function. It is normally set to $e^{-\frac{\Delta f}{T}}$, where $T$ is a control parameter, which corresponds to the temperature in analogy with the physical annealing. This acceptance function implies that the small increase in $f$ is more likely to be accepted than a large increase in $f$. When $T$ is high most uphill moves are accepted, but as $T$ approaches to zero, most uphill moves will be rejected. Therefore, SA algorithm starts with a high temperature to avoid being trapped in local minimum and proceeds by attempting a certain number of moves at each temperature by decreasing the temperature. Thus, the configuration decisions in SA proceed in a logical order.

The overall SA process steps are summarized as follows:

STEP 1 Select an initial temperature $T$.

STEP 2 Select a randomly generated initial solution vector, $X$, and generate the objective function.

STEP 3 Select the temperature change counter

STEP 4 Select a temperature cooling schedule

STEP 5 Select a repetition schedule that defines the number of iterations executed at each temperature

STEP 6 Give a random perturbation on $X$ and generate a new solution vector, $Y$, in the neighbourhood of current solution vector, $X$, evaluate the objective function

STEP 7 If $f(Y)<f(X)$, replace $X$ with $Y$. Update the existing optimal solution and go to Step 9.

STEP 8 Else accept $Y$ with the probability $p=e^{-\frac{\Delta f}{T}}$ where $\Delta f=f(Y)-f(X)$. If the solution is accepted, replace $X$ with $Y$. Update the existing optimal solution and go to Step 9.

STEP 9 Decrease the temperature periodically.

STEP 10 Repeat Steps 6 -9 until stopping criterion is met. 


\section{Simulated Annealing with Clone Operator}

In a previous work [27], we introduced a new operator on a population called Clone Operator. Cloning is carried out with a clone probability. The genes which are not cloned based on probability are mutated $(0 \rightarrow 1,1 \rightarrow 0)$. It is expected that the new member will have better qualities of the parent member. As an example, consider the string

\begin{tabular}{|l|l|l|l|l|l|l|l|l|l|}
\hline 1 & 0 & 1 & 1 & 1 & 0 & 1 & 0 & 0 & 1 \\
\hline
\end{tabular}

On applying the operator, each bit may undergo a cloning with a certain probability. If the cloning probability is 0.8 , two genes of the string will be mutated randomly and we might get, after cloning, the string-

\begin{tabular}{|l|l|l|l|l|l|l|l|l|l|}
\hline 1 & 1 & 1 & 1 & 1 & 0 & 0 & 0 & 0 & 1 \\
\hline
\end{tabular}

in which two genes, viz. the second and the seventh, are changed while all the others are cloned.

We propose the clone operator to generate a candidate solution vector for the solution vector in simulated annealing algorithm. The step by step procedure of the new algorithm, which we call SAC, can be summarized as follows:

STEP 1 Select an initial temperature $T$.

STEP 2 Select a randomly generated binary coded initial solution vector, $X$, of and generate the objective function.

STEP 3 Select the temperature change counter.

STEP 4 Select a temperature cooling schedule.

STEP 5 Select a repetition schedule that defines the number of iterations executed at each temperature.

STEP 6 Generate a new solution vector, $Y$, by applying clone operator on $X$ and evaluate the objective function.

STEP 7 If $f(Y)<f(X)$, replace $X$ with $Y$. Update the existing optimal solution and go to Step 9.

STEP 8 Else accept $Y$ with the probability $p=e^{-\frac{\Delta f}{T}}$ where $\Delta f=f(Y)-f(X)$. If the solution is accepted, replace $X$ with $Y$. Update the existing optimal solution and go to Step 9.

STEP 9 Decrease the temperature periodically.

STEP 10 Repeat Steps 6-9 until stopping criterion is met.

Fig.1 shows the schematic of the application of SAC algorithm. 


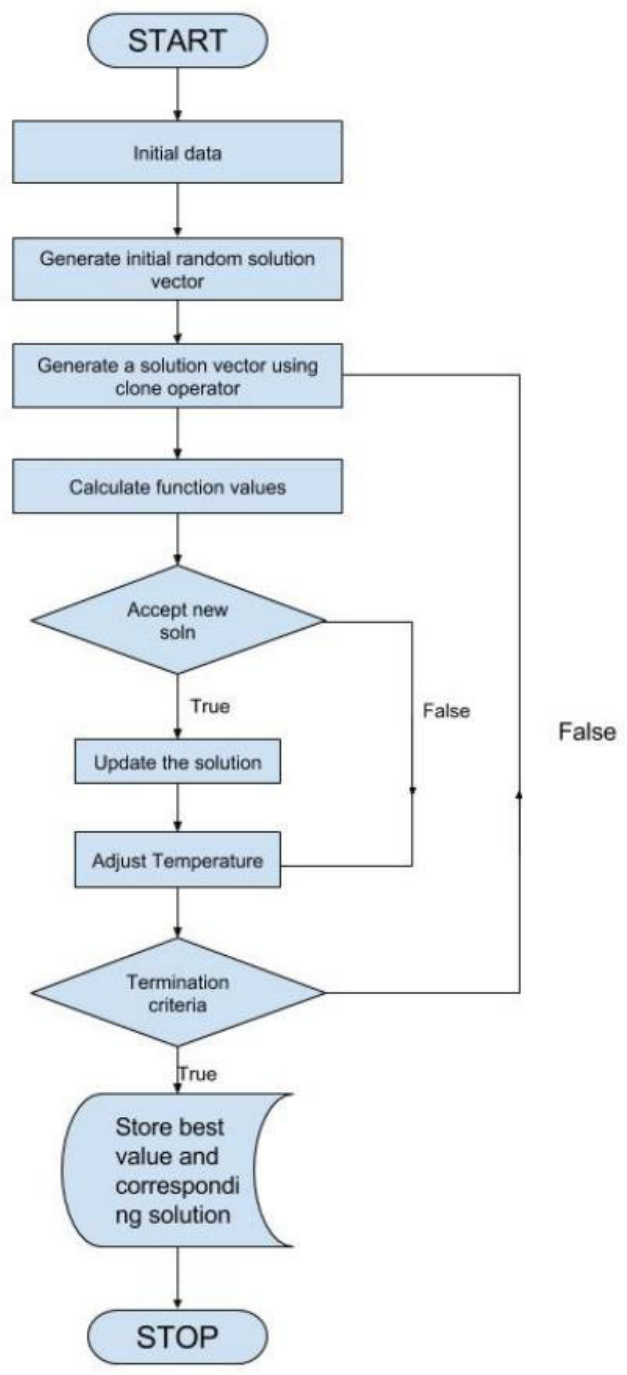

Figure 1: Flowchart for SAC algorithm

\section{Case studies}

We evaluate the performance of the proposed SAC algorithm on a set of 15 benchmark test functions (see table 1 and table 2) in the literature with different complexity. Experiments are conducted on the $2-D$ problems. The results of SAC on $2-D$ problems is compared with simple SA. Parameters used in the implementation of SAC and SA are : Bit length for one variable $=16$, probability of clone $=0.6$, the initial temperature $=1 / 10 / 100 / 1000$ according to the problem, temperature at which to stop $=e^{-8}$, maximum number of consecutive rejections $=1000$, maximum number of tries within one temperature $=300$, maximum number of successes within one temperature $=20$. 

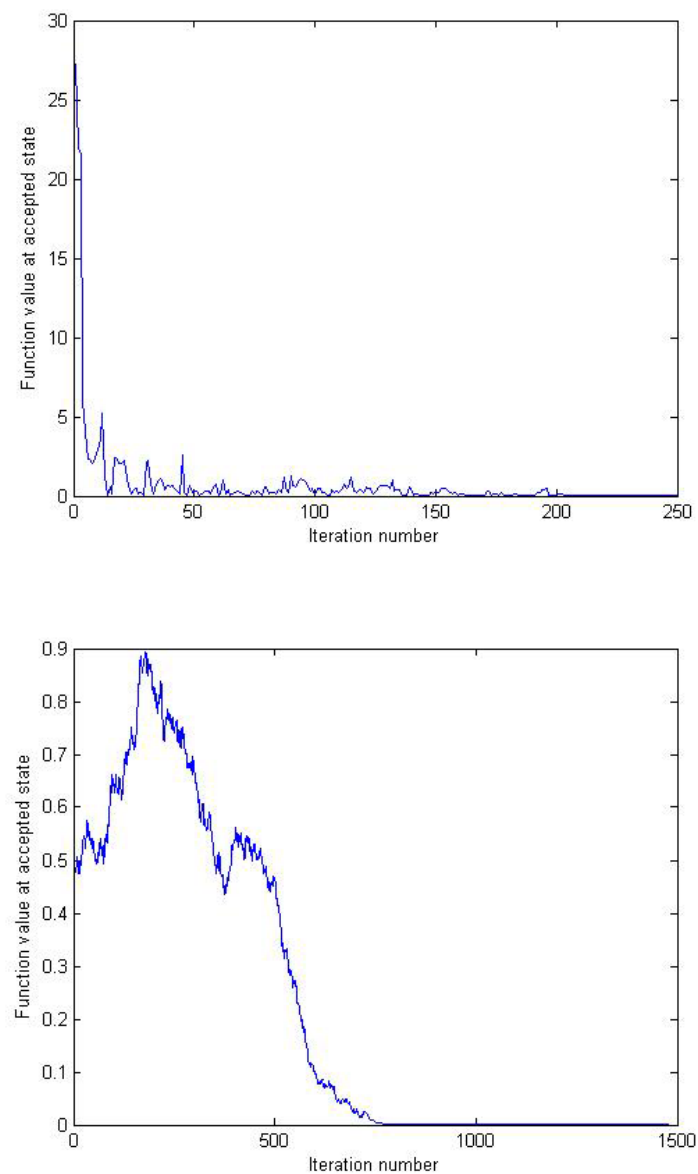

Figure 2: Convergence Graph for the function $g_{1}$ using SAC and SA algorithms. Left plot is for SAC algorithm and right plot is for SA algorithm.

We use the MATLAB to implement the algorithms. All the implementations were developed using a computer with processor Core 2 Quad processor of 64 bits that works to a frequency of $2.7 \mathrm{GHz}, 4 \mathrm{~GB}$ of RAM Memory and Windows XP Professional Version.

In table 3 , we can find the experimental results. The table 2 provides the results with 50 Monte-Carlo simulations with the changes in the random sequence. The results are provided in terms of statistical parameters such as average, the median, the best value, the worst value and standard deviation obtained for each test functions. The number of function evaluations for convergence for best and the average number of function evalutions taken by the two methods are also presented. The convergence maps of the algorithm on SAC and SA functions $g_{1}$ to $g_{15}$ are plotted in figures 1-15 respectively. 
Table 1: Test functions $g_{1}$ to $g_{12}$

\begin{tabular}{|c|c|}
\hline Function & Definition \\
\hline$g_{1}$ & $\begin{array}{l}g_{1}: \text { Sphere function(De Jong's function 1) } \\
\qquad g_{1}(x)=\sum_{i=1}^{D} x_{i}^{2} \\
-5.12 \leq x_{i} \leq 5.12, \quad x *=(0,0, \ldots 0), \quad g_{1}(x *)=0\end{array}$ \\
\hline$g_{2}$ & $\begin{array}{l}g_{2} \text { : Rosenbrock function (De Jong's function } 2 \text {, Banana function) } \\
\qquad g_{2}(x)=\sum_{i=1}^{D-1}\left[100\left(x_{i+1}-x_{i}^{2}\right)^{2}+\left(x_{i}-1\right)^{2}\right] \\
-2.048 \leq x_{i} \leq 2.048, \quad x *=(1,1, \ldots 1), \quad g_{2}(x *)=0\end{array}$ \\
\hline$g_{3}$ & $\begin{array}{l}g_{3} \text { :Ackley's function } \\
g_{3}(x)=20+e-20 \exp \left(-0.2 \sqrt{\frac{1}{D} \sum_{i=1}^{D} x_{i}^{2}}\right)-\exp \left(\frac{1}{D} \sum_{i=1}^{D} \cos \left(2 \pi x_{i}\right)\right) \\
-32.768 \leq x_{i} \leq 32.768, \quad x *=(0,0, \ldots 0), \quad g_{3}(x *)=0\end{array}$ \\
\hline$g_{4}$ & $\begin{array}{l}g_{4} \text { : Rastrigin function } \\
\qquad g_{4}(x)=10 D+\sum_{i=1}^{D} x_{i}^{2}-10 \cos \left(2 \pi x_{i}\right) \\
-5.12 \leq x_{i} \leq 5.12, \quad x *=(0,0, \ldots 0), \quad g_{4}(x *)=0\end{array}$ \\
\hline$g_{5}$ & $\begin{array}{l}g_{5}: \text { : Griewank function } \\
\qquad g_{5}(x)=1+\sum_{i=1}^{D} \frac{x_{i}^{2}}{4000}-\prod_{i=1}^{D} \cos \left(\frac{x_{i}}{\sqrt{i}}\right) \\
-600 \leq x_{i} \leq 600, \quad x *=(0,0, \ldots 0), \quad g_{5}(x *)=0\end{array}$ \\
\hline$g_{6}$ & $\begin{array}{l}g_{6} \text { : Schwefel's Double Sum function } \\
\qquad g_{6}(x)=\sum_{i=1}^{D}\left(\sum_{j=1}^{i} x_{j}\right)^{2} \\
-65.536 \leq x_{i} \leq 65.536, \quad x *=(0,0, \ldots 0), \quad g_{6}(x *)=0\end{array}$ \\
\hline
\end{tabular}


Table 1: Continued.

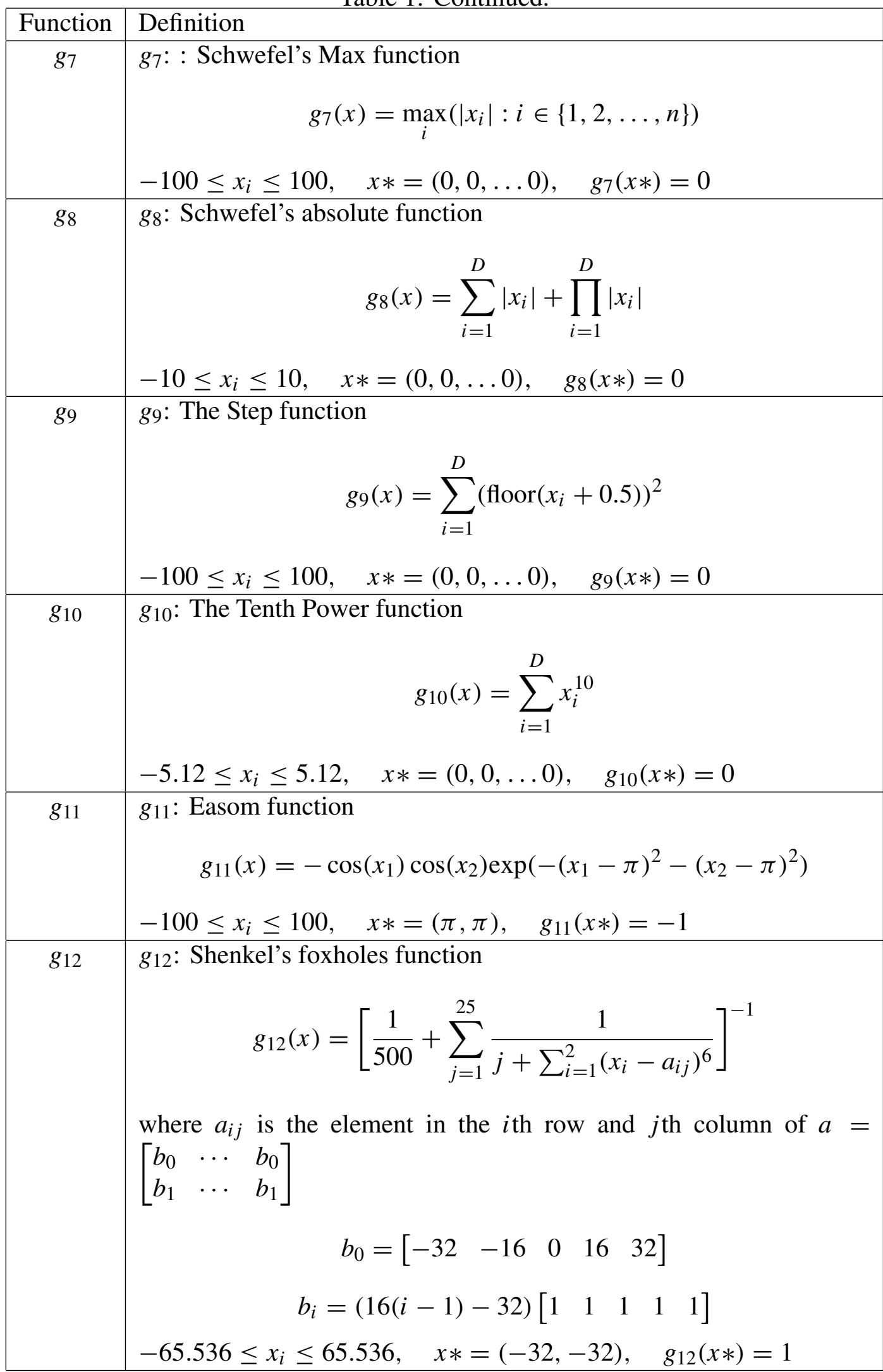


Table 2: Test functions $g_{13}$ to $g_{15}$

\begin{tabular}{|c|c|}
\hline Function & Definition \\
\hline \multirow[t]{4}{*}{$g_{13}$} & $g_{13}:$ Goldstein-Price function \\
\hline & $g_{13}(x)=\left[1+\left(x_{1}+x_{2}+1\right)^{2}\left(19-14 x_{1}+3 x_{1}^{2}-14 x_{2}+6 x_{1} x_{2}+3 x_{2}^{2}\right)\right]$ \\
\hline & $\times\left[30+\left(2 x_{1}-3 x_{2}\right)^{2}\left(18-32 x_{1}+12 x_{1}^{2}+48 x_{2}-36 x_{1} x_{2}+27 x_{2}^{2}\right)\right]$ \\
\hline & $-2 \leq x_{i} \leq 2, \quad x *=(0,-1), \quad g_{13}(x *)=3$ \\
\hline \multirow[t]{3}{*}{$g_{14}$} & $g_{14}:$ Six hump camel back function \\
\hline & $g_{14}(x)=\left[\left(4-2.1 x_{1}^{2}+x_{1}^{4 / 3}\right) x_{1}^{2}+x_{1} x_{2}+\left(-4+4 x_{2}^{2}\right) x_{2}^{2}\right.$ \\
\hline & 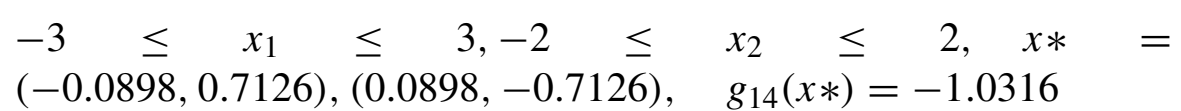 \\
\hline \multirow[t]{3}{*}{$g_{15}$} & $g_{15}:$ Branin's rcos function \\
\hline & $g_{15}(x)=\left(x_{2}-\frac{5.1}{4 \pi^{2}} x_{1}^{2}+\frac{5}{\pi} x_{1}-6\right)^{2}+\left(10-\frac{5}{4 \pi}\right) \cos x_{1}+10$ \\
\hline & 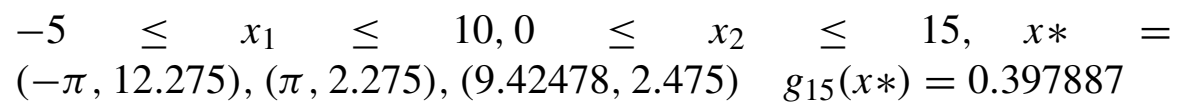 \\
\hline
\end{tabular}

Table 3: Comparison results of the best value, the median, the worst value, average, and standard deviation of the functions $g_{1}-g_{15}$ for 50 independent runs using CGA,CEA and GA methods

\begin{tabular}{|c|c|c|c|c|c|c|c|c|}
\hline $\mathrm{F}$ & Method & Best & Median & Worse & Average & S.D & NFCB & ANFC \\
\hline$g_{1}$ & SAC & $1.1642 \mathrm{E}-08$ & $2.2935 \mathrm{E}-06$ & $3.9233 \mathrm{E}-06$ & $2.2972 \mathrm{E}-06$ & $1.3876 \mathrm{E}-06$ & 12063 & 11492 \\
\cline { 2 - 9 } & SA & $1.4136 \mathrm{E}-10$ & $1.1834 \mathrm{E}-08$ & $4.9742 \mathrm{E}-08$ & $1.2281 \mathrm{E}-09$ & $7.1842 \mathrm{E}-06$ & 4372 & 5132 \\
\hline \multirow{2}{*}{$g_{2}$} & SAC & $2.3516 \mathrm{E}-08$ & $7.7233 \mathrm{E}-06$ & $1.3587 \mathrm{E}-05$ & $7.4902 \mathrm{E}-06$ & $4.3857 \mathrm{E}-06$ & 13209 & 12690 \\
\cline { 2 - 9 } & SA & $1.8498 \mathrm{E}-06$ & $8.1437 \mathrm{E}-06$ & $5.4235 \mathrm{E}-05$ & $9.8367 \mathrm{E}-06$ & $4.0757 \mathrm{E}-05$ & 10651 & 11770 \\
\hline \multirow{2}{*}{$g_{3}$} & SAC & 0.0020 & 0.0083 & 0.0130 & 0.0082 & 0.0032 & 3300 & 4092 \\
\cline { 2 - 9 } & SA & $9.7133 \mathrm{E}-07$ & $4.4241 \mathrm{E}-05$ & $7.4176 \mathrm{E}-04$ & $1.7511 \mathrm{E}-05$ & $3.2534 \mathrm{E}-05$ & 11959 & 11554 \\
\hline \multirow{2}{*}{$g_{4}$} & SAC & $2.3097 \mathrm{E}-06$ & $1.5013 \mathrm{E}-04$ & $3.3490 \mathrm{E}-04$ & $1.6454 \mathrm{E}-04$ & $1.1561 \mathrm{E}-04$ & 8386 & 6749 \\
\cline { 2 - 9 } & SA & $2.6316 \mathrm{E}-08$ & $1.6317 \mathrm{E}-07$ & $1.6523 \mathrm{E}-06$ & $4.6412 \mathrm{E}-07$ & $1.8557 \mathrm{E}-05$ & 10216 & 11486 \\
\hline \multirow{2}{*}{$g_{5}$} & SAC & $5.6607 \mathrm{E}-04$ & 0.0071 & 0.0082 & 0.0057 & 0.0025 & 6805 & 4945 \\
\cline { 2 - 10 } & SA & 0.2929 & 0.2929 & 0.2929 & 0.2929 & $1.0121 \mathrm{E}-17$ & 4036 & 4632 \\
\hline \multirow{2}{*}{$g_{6}$} & SAC & $9.8374 \mathrm{E}-07$ & $1.3477 \mathrm{E}-04$ & $2.7643 \mathrm{E}-04$ & $1.4201 \mathrm{E}-04$ & $7.9812 \mathrm{E}-05$ & 12628 & 12182 \\
\cline { 2 - 10 } & SA & $3.5179 \mathrm{E}-09$ & $6.1342 \mathrm{E}-09$ & $2.9201 \mathrm{E}-07$ & $9.3266 \mathrm{e}-08$ & $1.1271 \mathrm{e}-05$ & 5826 & 6164 \\
\hline \multirow{2}{*}{$g_{7}$} & SAC & 0.0015 & 0.0107 & 0.0137 & 0.0091 & 0.0034 & 7980 & 6496 \\
\cline { 2 - 9 }$y$ & SA & $3.2158 \mathrm{E}-06$ & $5.1157 \mathrm{E}-05$ & $4.9007 \mathrm{E}-05$ & $1.0211 \mathrm{E}-05$ & $1.0193 \mathrm{E}-05$ & 9288 & 9476 \\
\hline \multirow{2}{*}{$g_{8}$} & SAC & $3.0520 \mathrm{E}-04$ & 0.0012 & 0.0021 & 0.0014 & $5.5128 \mathrm{E}-04$ & 7462 & 8519 \\
\cline { 2 - 9 } & SA & $1.9585 \mathrm{E}-07$ & $2.6243 \mathrm{E}-06$ & $6.1208 \mathrm{E}-06$ & $3.1147 \mathrm{E}-06$ & $3.1206 \mathrm{E}-05$ & 7384 & 8134 \\
\hline \multirow{2}{*}{$g_{9}$} & SAC & 0 & 0 & 0 & 0 & 0 & 3000 & 2448 \\
\cline { 2 - 9 } & SA & 0 & 0 & 0 & 0 & 0 & 1710 & 2236 \\
\hline
\end{tabular}


Table 3: Continued.

\begin{tabular}{|c|c|c|c|c|c|c|c|c|}
\hline $\mathrm{F}$ & Method & Best & Median & Worse & Average & S.D & NFCB & ANFC \\
\hline$g_{10}$ & SAC & $3.7755 \mathrm{E}-33$ & $1.0886 \mathrm{E}-21$ & $6.9026 \mathrm{E}-20$ & $8.7412 \mathrm{E}-21$ & $1.8272 \mathrm{E}-20$ & 17212 & 17922 \\
\cline { 2 - 9 } & SA & $3.6763 \mathrm{E}-14$ & $1.3712 \mathrm{E}-14$ & $7.6102 \mathrm{E}-13$ & $4.7126 \mathrm{e}-14$ & $3.1773 \mathrm{E}-05$ & 2334 & 1376 \\
\hline \multirow{2}{*}{$g_{11}$} & SAC & -1.0000 & -0.9997 & -0.9992 & -0.9996 & $2.5179 \mathrm{E}-04$ & 4341 & 4234 \\
\cline { 2 - 9 } & SA & -1 & -1 & -0.9997 & -1 & $7.1214 \mathrm{E}-05$ & 3887 & 4237 \\
\hline \multirow{2}{*}{$g_{12}$} & SAC & 0.9980 & 0.9980 & 0.9980 & 0.9980 & $3.9318 \mathrm{E}-10$ & 10867 & 16128 \\
\cline { 2 - 9 } & SA & 0.9980 & 0.9980 & 0.9980 & 0.9980 & $1.2305 \mathrm{E}-16$ & 6215 & 6186 \\
\hline \multirow{2}{*}{$g_{13}$} & SAC & 3.0000 & 3.0000 & 3.0001 & 3.0000 & $1.9365 \mathrm{E}-05$ & 3000 & 6516 \\
\cline { 2 - 9 } & SA & 3.0000 & 3.0000 & 3.0000 & 3.0000 & $1.2614 \mathrm{E}-05$ & 8967 & 9154 \\
\hline \multirow{2}{*}{$g_{14}$} & SAC & -1.0316 & -1.0316 & -1.0316 & -1.0316 & $3.3893 \mathrm{E}-07$ & 10030 & 11387 \\
\cline { 2 - 9 } & SA & -1.0316 & -1.0316 & -1.0316 & -1.0316 & $1.2164 \mathrm{E}-08$ & 6088 & 6854 \\
\hline \multirow{2}{*}{$g_{15}$} & SAC & 0.397887 & 0.397887 & 0.3978869 & 0.397887 & $1.7308 \mathrm{E}-06$ & 9298 & 12389 \\
\cline { 2 - 9 } & SA & 0.397887 & 0.397887 & 0.397887 & 0.397887 & $1.1699 \mathrm{E}-06$ & 7372 & 7895 \\
\hline
\end{tabular}

F-Function NFCB-Number of function evaluation for the convergence of best ANFC-Average number of function evaluation for the convergence
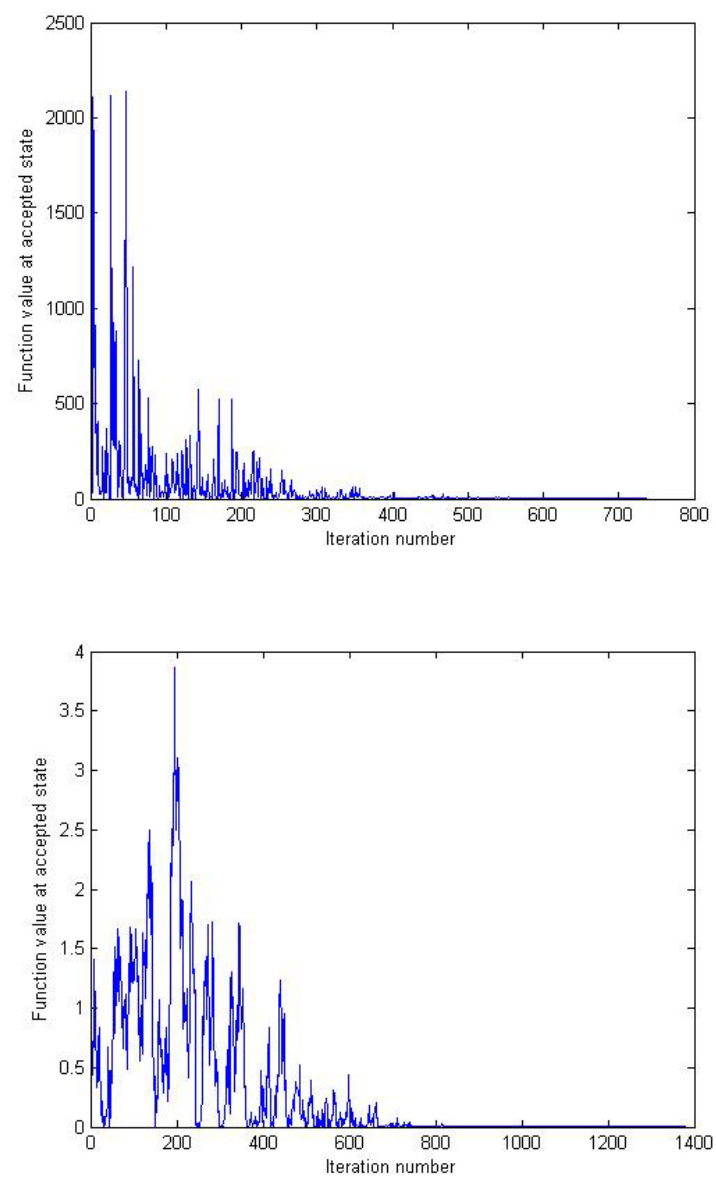

Figure 3: Convergence Graph for the function $g_{2}$ using SAC and SA algorithms. Left plot is for SAC algorithm and right plot is for SA algorithm. 

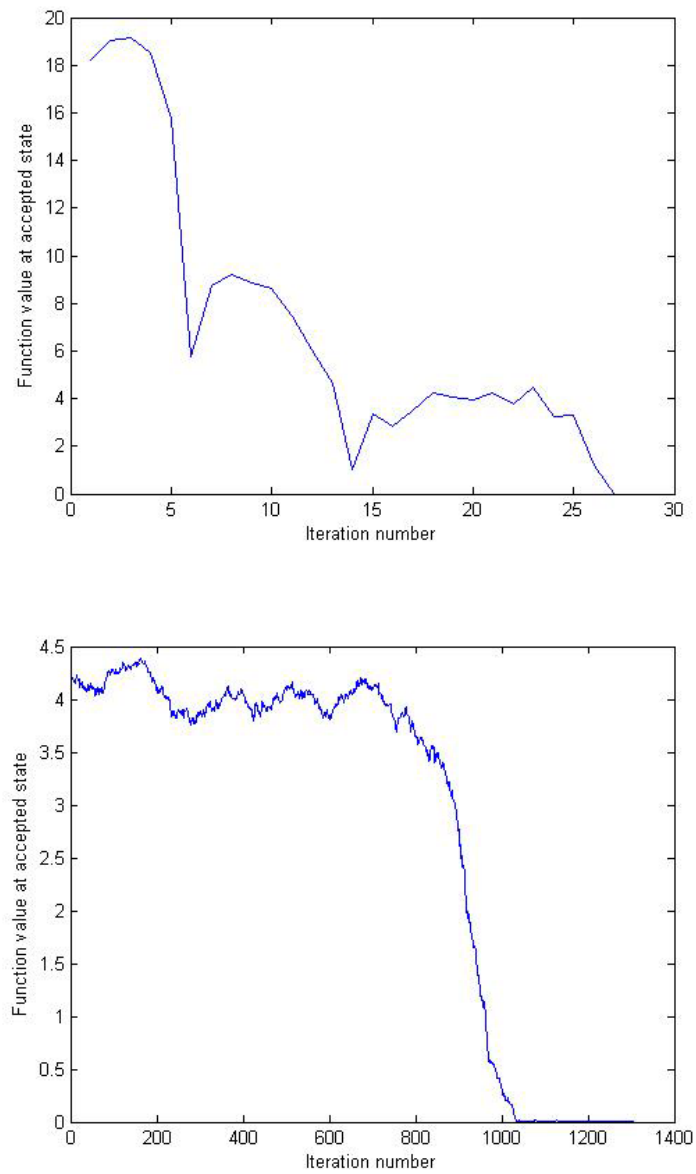

Figure 4: Convergence Graph for the function $g_{3}$ using SAC and SA algorithms. Left plot is for SAC algorithm and right plot is for SA algorithm. 

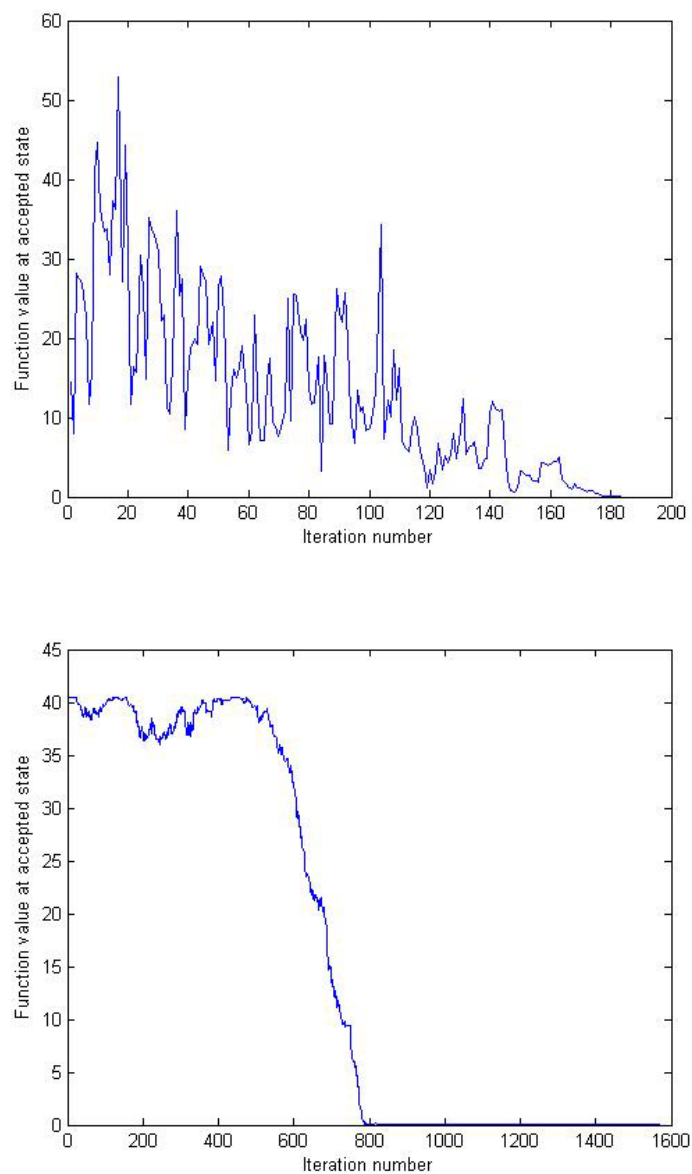

Figure 5: Convergence Graph for the function $g_{4}$ using SAC and SA algorithms. Left plot is for SAC algorithm and right plot is for SA algorithm. 

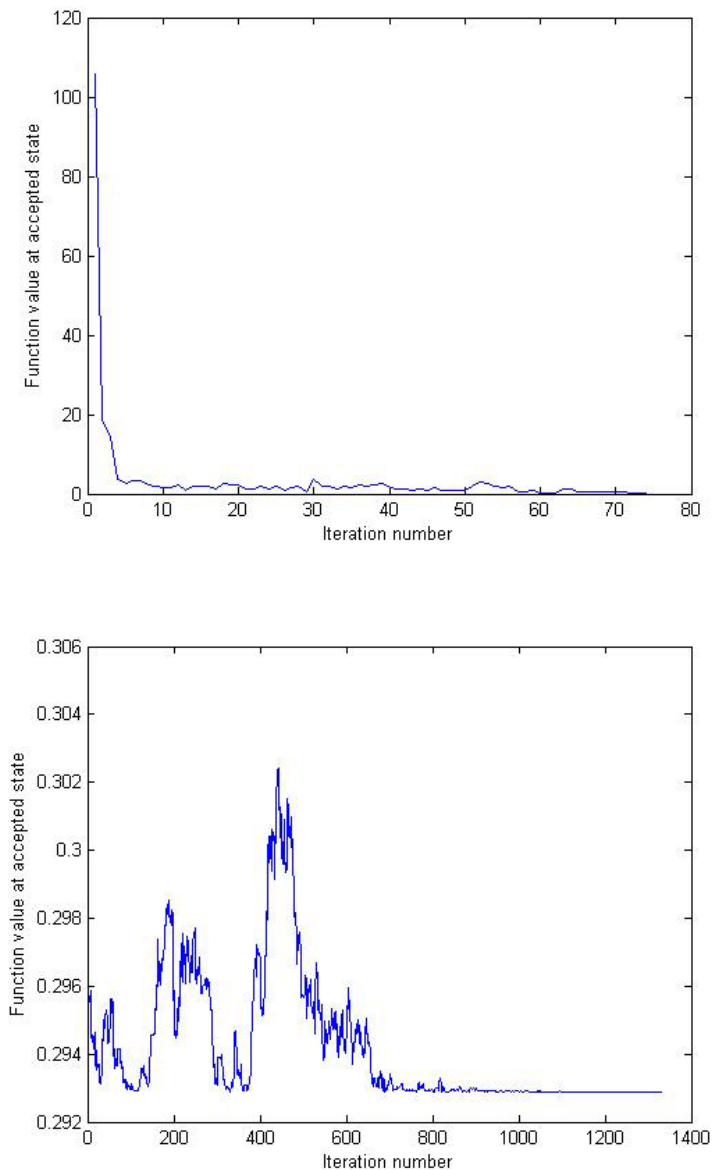

Figure 6: Convergence Graph for the function $g_{5}$ using SAC and SA algorithms. Left plot is for SAC algorithm and right plot is for SA algorithm. 

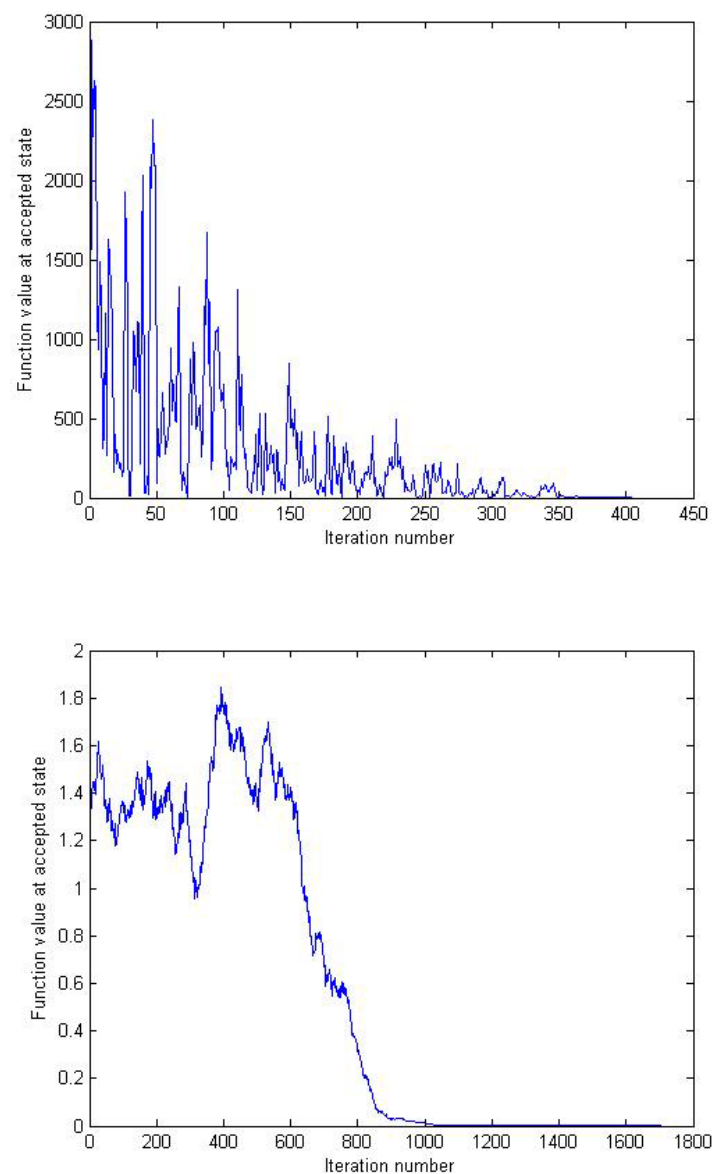

Figure 7: Convergence Graph for the function $g_{6}$ using SAC and SA algorithms. Left plot is for SAC algorithm and right plot is for SA algorithm. 

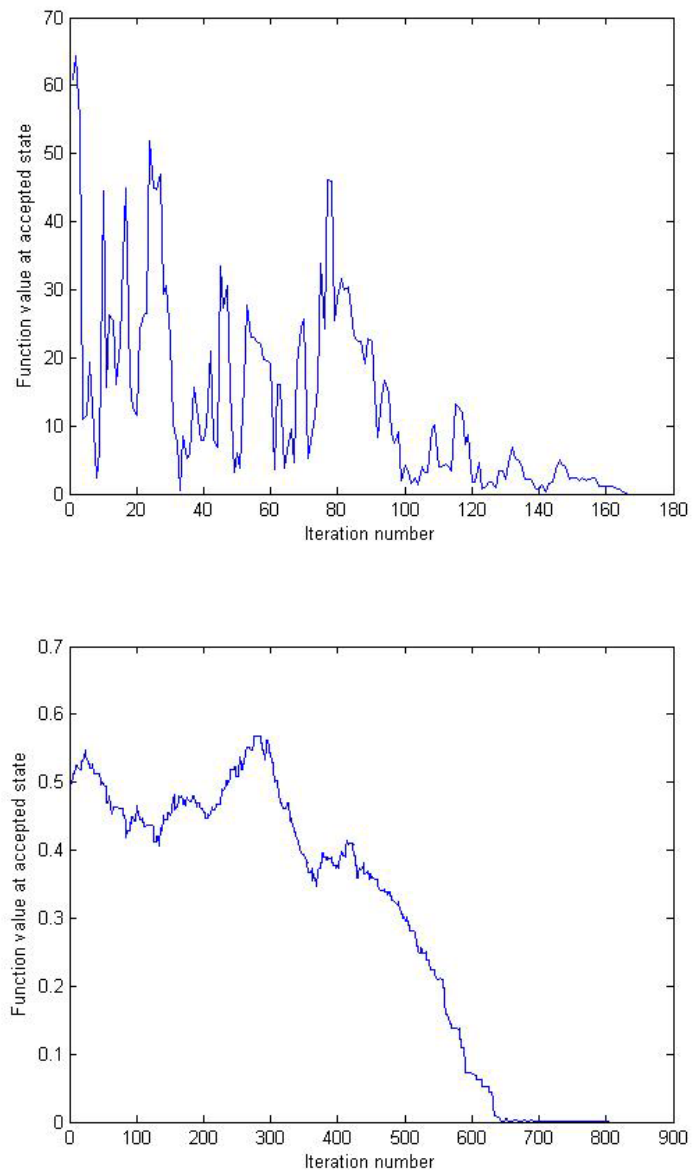

Figure 8: Convergence Graph for the function $g_{7}$ using SAC and SA algorithms. Left plot is for SAC algorithm and right plot is for SA algorithm. 

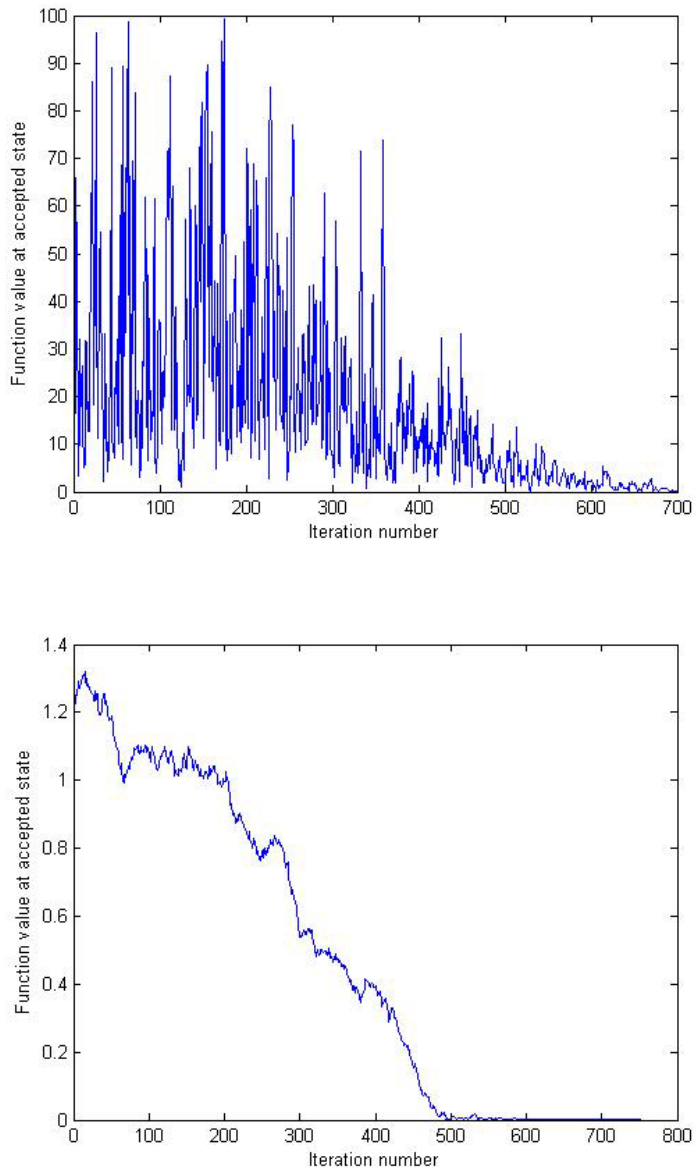

Figure 9: Convergence Graph for the function $g_{8}$ using SAC and SA algorithms. Left plot is for SAC algorithm and right plot is for SA algorithm. 

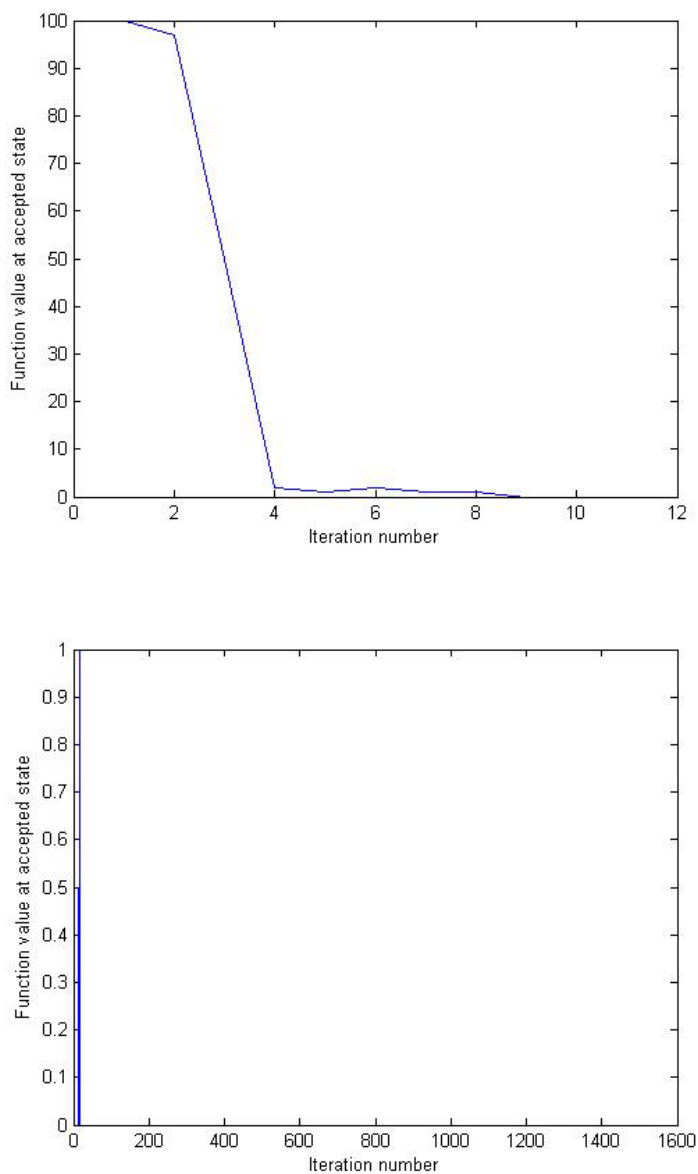

Figure 10: Convergence Graph for the function $g_{9}$ using SAC and SA algorithms. Left plot is for SAC algorithm and right plot is for SA algorithm. 

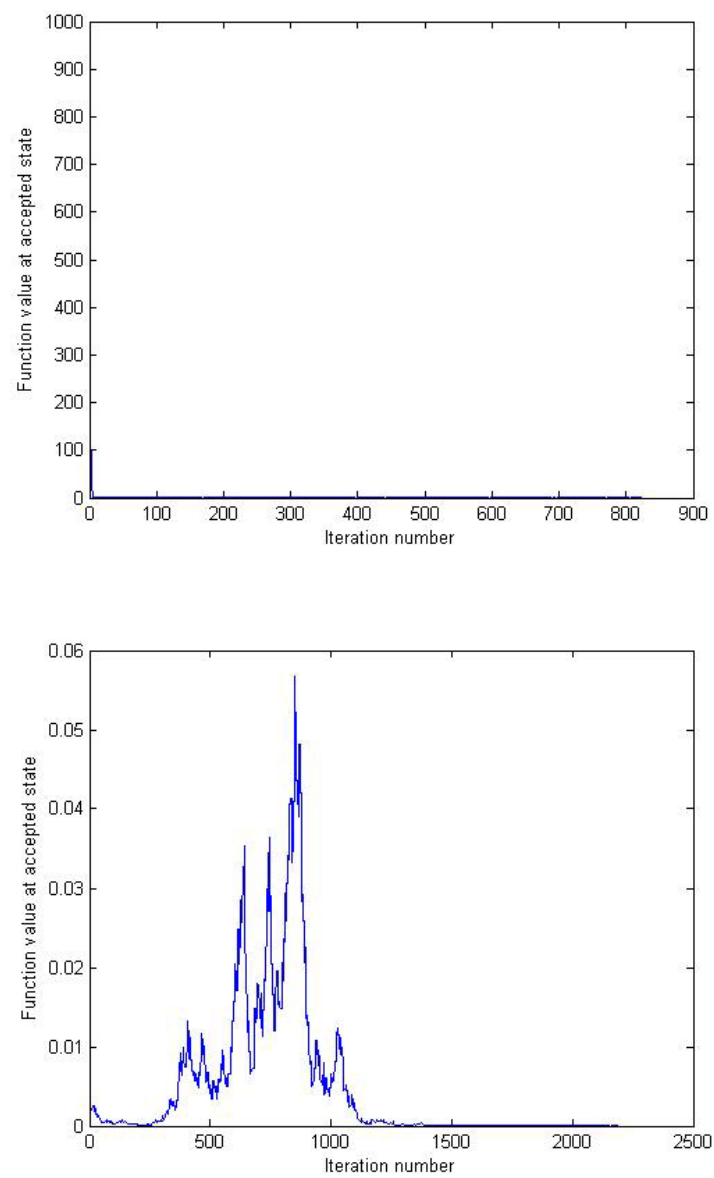

Figure 11: Convergence Graph for the function $g_{10}$ using SAC and SA algorithms. Left plot is for SAC algorithm and right plot is for SA algorithm. 

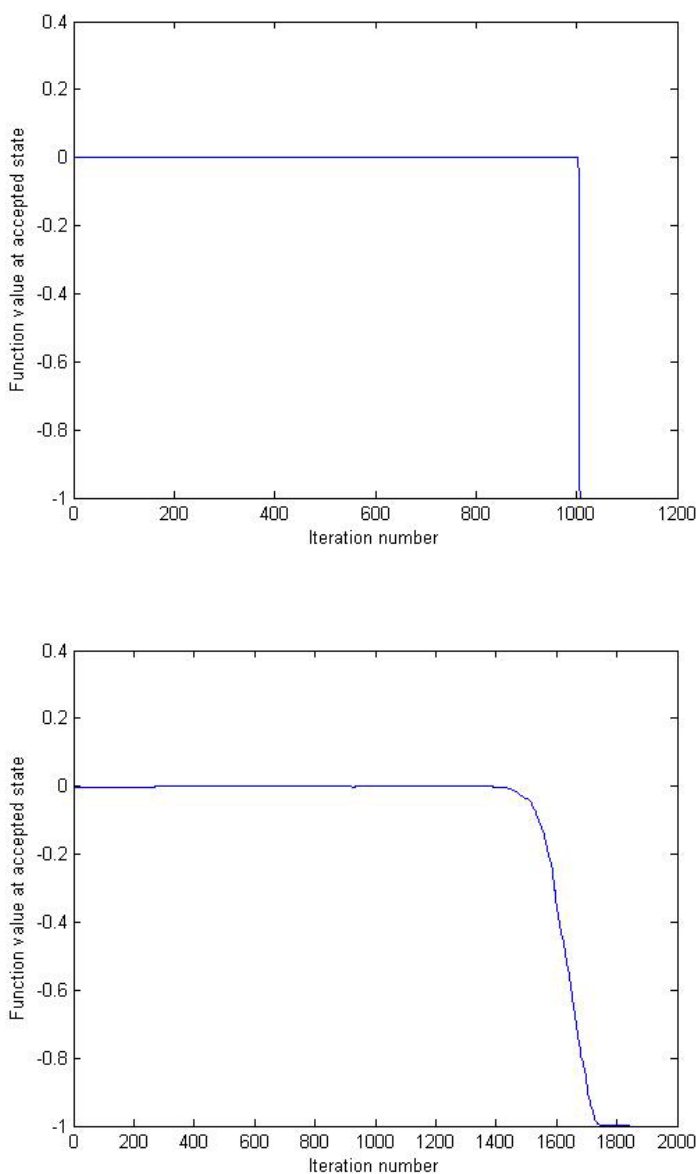

Figure 12: Convergence Graph for the function $g_{11}$ using SAC and SA algorithms. Left plot is for SAC algorithm and right plot is for SA algorithm. 

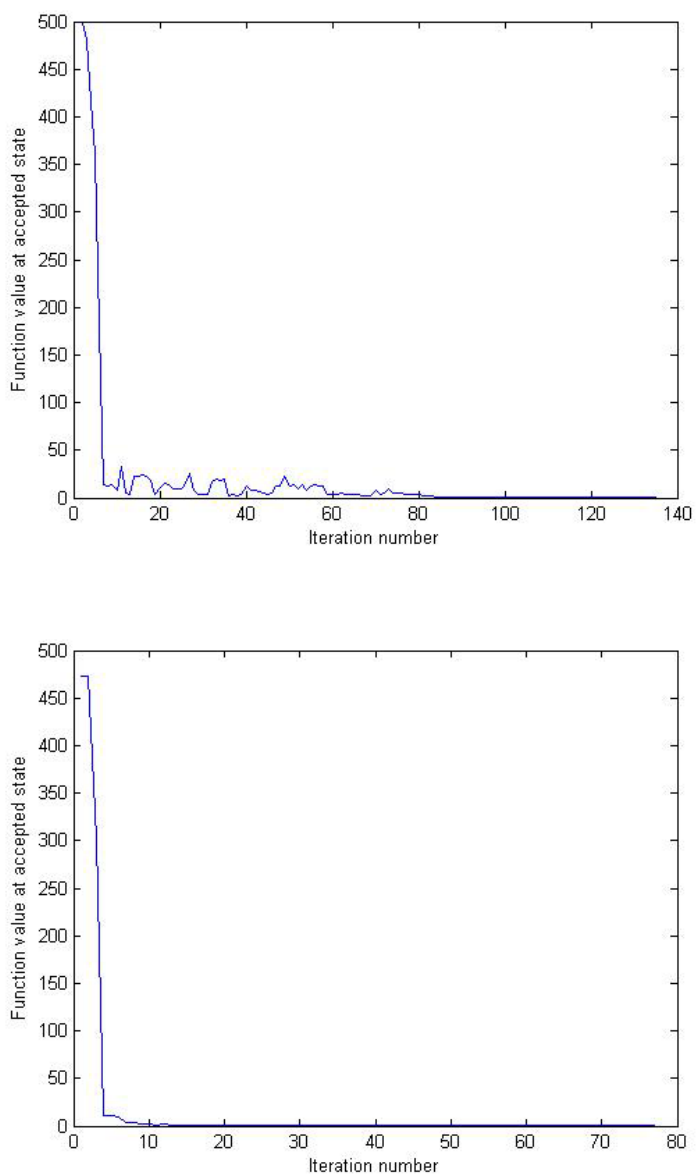

Figure 13: Convergence Graph for the function $g_{12}$ using SAC and SA algorithms. Left plot is for SAC algorithm and right plot is for SA algorithm. 

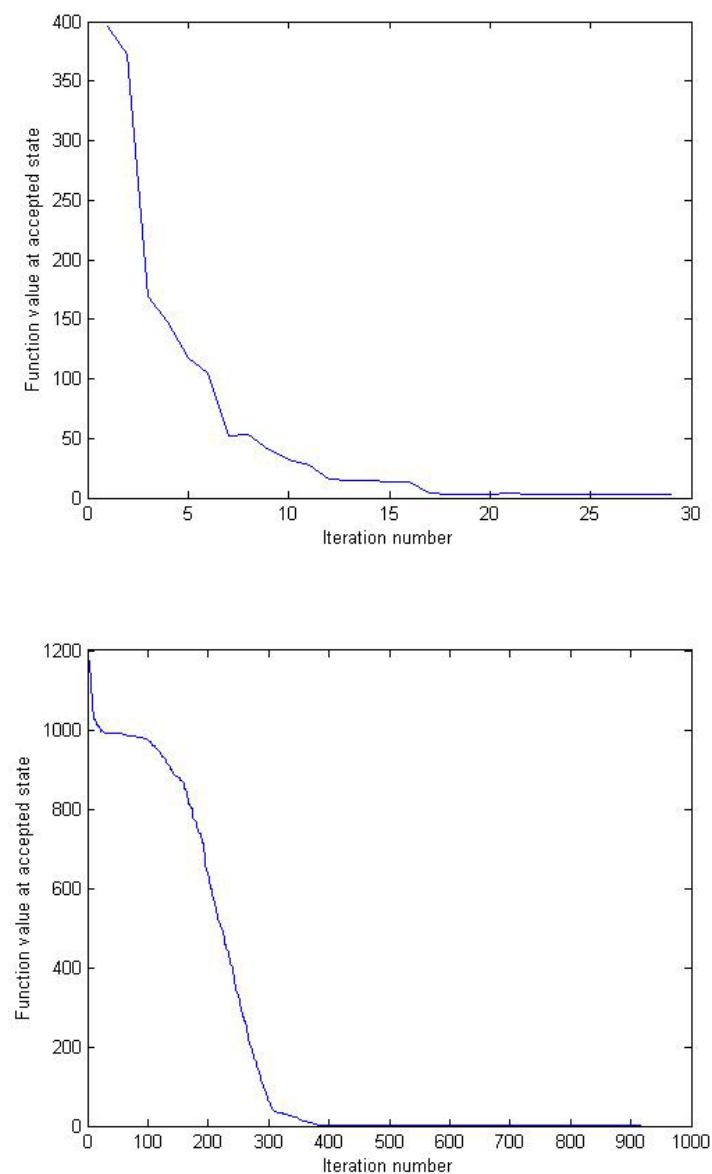

Figure 14: Convergence Graph for the function $g_{13}$ using SAC and SA algorithms. Left plot is for SAC algorithm and right plot is for SA algorithm. 

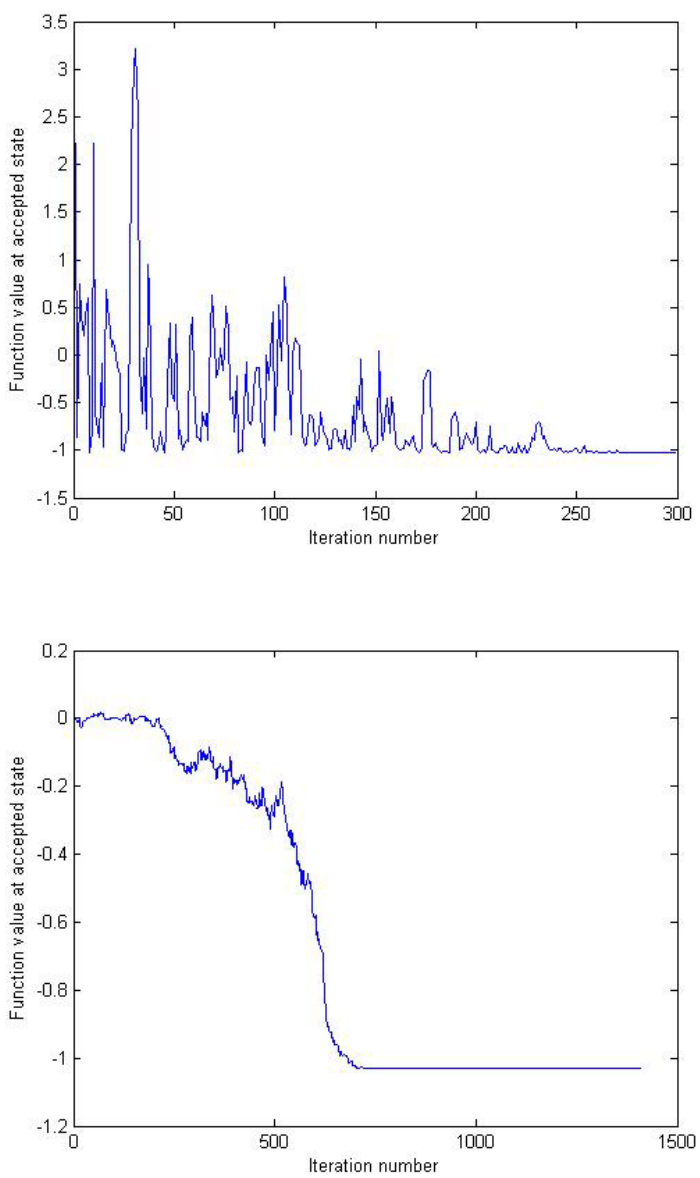

Figure 15: Convergence Graph for the function $g_{14}$ using SAC and SA algorithms. Left plot is for SAC algorithm and right plot is for SA algorithm. 

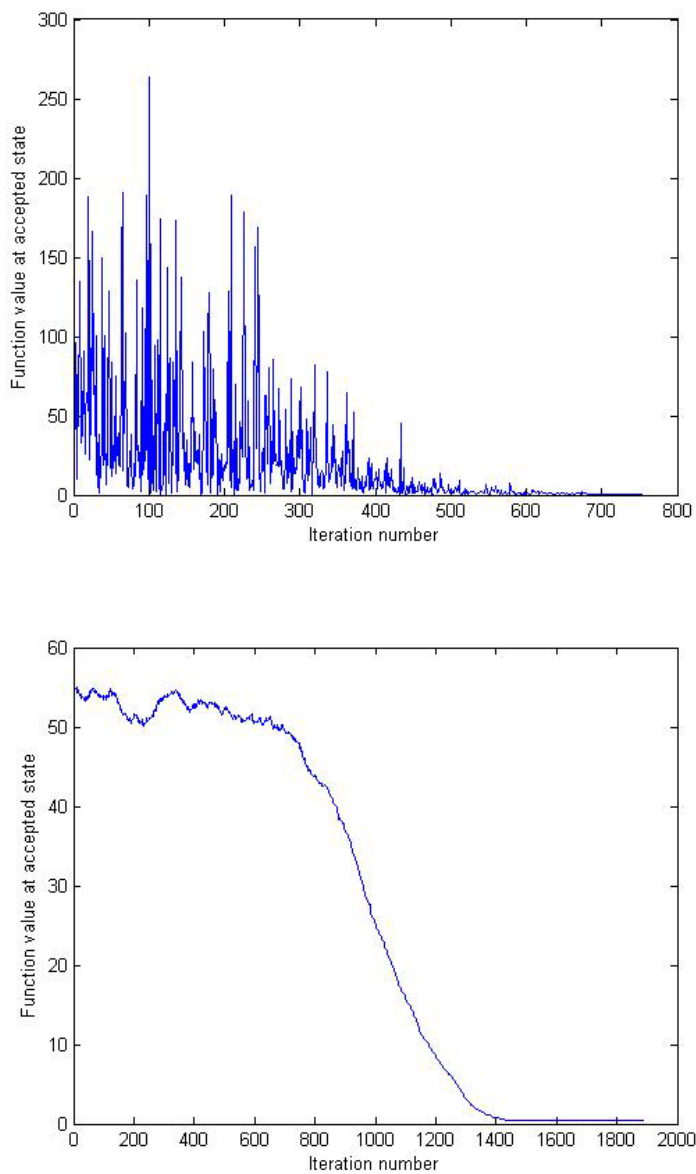

Figure 16: Convergence Graph for the function $g_{15}$ using SAC and SA algorithms. Left plot is for SAC algorithm and right plot is for SA algorithm.

\section{Conclusion}

In this paper a clone operator which imitates the biological cloning process to generate a candidate solution vector in simulated annealing algorithm is proposed. We have simulated the results for the SA algorithm and SA with clone operator with the help of various case studies obtained from literature. The results of the two methods, namely proposed SAC method and SA, indicate that the proposed SAC method is capable of generating better solution quality.

\section{Acknowledgment}

The authors would like to thank Abhay Kumar, Group Director AFDG, S. Pandian, Deputy Director AERO and K. Sivan, Director VSSC for encouragement and support and also for providing the computational facilities. 


\section{References}

[1] S. Kirkpatrick, C. D. Gelatt, M. P. Vecchi et al., "Optimization by simulated annealing," Science, vol. 220, no. 4598, pp. 671-680, 1983.

[2] V. Černỳ, "Thermodynamical approach to the traveling salesman problem: An efficient simulation algorithm," Journal of optimization theory and applications, vol. 45, no. 1, pp. 41-51, 1985.

[3] N. Metropolis, A. W. Rosenbluth, M. N. Rosenbluth, A. H. Teller, and E. Teller, "Equation of state calculations by fast computing machines," The journal of chemical physics, vol. 21, no. 6, pp. 1087-1092, 1953.

[4] D. Henderson, S. H. Jacobson, and A. W. Johnson, "The theory and practice of simulated annealing," in Handbook of metaheuristics. Springer, 2003, pp. 287319.

[5] E. H. Aarts and J. H. Korst, "Simulated annealing," ISSUES, vol. 1, p. 16, 1988.

[6] A. G. Nikolaev and S. H. Jacobson, "Simulated annealing," in Handbook of metaheuristics. Springer, 2010, pp. 1-39.

[7] K. A. Dowsland and J. M. Thompson, "Simulated annealing," in Handbook of natural computing, Springer, 2012, pp. 1623-1655.

[8] F. Romeo and A. Sangiovanni-Vincentelli, "A theoretical framework for simulated annealing," Algorithmica, vol. 6, no. 1, pp. 302-345, 1991.

[9] M. Fleischer, "Simulated annealing: past, present, and future," in Simulation Conference Proceedings, 1995. Winter. IEEE, 1995, pp. 155-161.

[10] B. Suman and P. Kumar, "A survey of simulated annealing as a tool for single and multiobjective optimization," Journal of the operational research society, vol. 57, no. 10, pp. 1143-1160, 2006.

[11] P. J. Laarhoven, Theoretical and computational aspects of simulated annealing. Centrum voor wiskunde en informatica, 1988, vol. 51.

[12] V. Fabian, "Simulated annealing simulated," Computers \& Mathematics with Applications, vol. 33, no. 1-2, pp. 81-94, 1997.

[13] I. O. Bohachevsky, M. E. Johnson, and M. L. Stein, "Generalized simulated annealing for function optimization," Technometrics, vol. 28, no. 3, pp. 209-217, 1986.

[14] M. Locatelli, "Convergence and first hitting time of simulated annealing algorithms for continuous global optimization," Mathematical Methods of Operations Research, vol. 54, no. 2, pp. 171-199, 2001.

[15] C. J. Bélisle, "Convergence theorems for a class of simulated annealing algorithms on d," Journal of Applied Probability, vol. 29, no. 04, pp. 885-895, 1992.

[16] B. Suman, "Multiobjective simulated annealing-a metaheuristic technique for multiobjective optimization of a constrained problem," Foundations of Computing and Decision Sciences, vol. 27, no. 3, pp. 171-191, 2002.

[17] _ _ "Simulated annealing-based multiobjective algorithms and their application for system reliability," Engineering Optimization, vol. 35, no. 4, pp. 391-416, 2003. 
[18] — "Study of self-stopping pdmosa and performance measure in multiobjective optimization," Computers \& Chemical Engineering, vol. 29, no. 5, pp. 1131-1147, 2005.

[19] C. J. Bélisle, H. E. Romeijn, and R. L. Smith, "Hit-and-run algorithms for generating multivariate distributions," Mathematics of Operations Research, vol. 18, no. 2, pp. 255-266, 1993.

[20] B. Hajek, "Cooling schedules for optimal annealing," Mathematics of Operations Research, vol. 13, no. 2, pp. 311-329, 1988.

[21] Z. B. Zabinsky, R. L. Smith, J. F. McDonald, H. E. Romeijn, and D. E. Kaufman, "Improving hit-and-run for global optimization," Journal of Global Optimization, vol. 3, no. 2, pp. 171-192, 1993.

[22] S. Anily and A. Federgruen, "Simulated annealing methods with general acceptance probabilities," Journal of Applied Probability, vol. 24, no. 03, pp. 657-667, 1987.

[23] D. Mitra, F. Romeo, and A. Sangiovanni-Vincentelli, "Convergence and finite-time behavior of simulated annealing," Advances in applied probability, vol. 18, no. 3, pp. 747-771, 1986.

[24] M. Lundy and A. Mees, "Convergence of an annealing algorithm," Mathematical programming, vol. 34, no. 1, pp. 111-124, 1986.

[25] B. Gidas, "Nonstationary markov chains and convergence of the annealing algorithm," Journal of Statistical Physics, vol. 39, no. 1-2, pp. 73-131, 1985.

[26] S. B. Gelfand and S. K. Mitter, "Analysis of simulated annealing for optimization," in Decision and Control, 1985 24th IEEE Conference on, vol. 24. IEEE, 1985, pp. 779-786.

[27] G. S. Biju and A. K. Anilkumar, "An integrated genetic algorithm with clone operator," International Journal of Pure and Applied Mathematical Sciences, vol. 9, no. 2, pp. 145-164, 2016. 